\title{
The use of action functionals within the quantum-like paradigm
}

\author{
Emmanuel Haven, Andrei Khrennikov* \\ School of Management and IQSCS, University of Leicester, UK \\ *International Center for Mathematical Modeling in Physics and Cognitive Sciences \\ Linnaeus University, Växjö, Sweden
}

\begin{abstract}
Arbitrage is a key concept in the theory of asset pricing and it plays a crucial role in financial decision making. The concept of the curvature of so called 'fibre bundles' can be used to define arbitrage. The concept of 'action' can play an important role in the definition of arbitrage. In this paper we connect the probabilities emerging from a (non) zero linear action with so called risk neutral probabilities. The paper also shows how arbitrage/non arbitrage can be well defined within a quantum-like paradigm. We also discuss briefly the behavioral dimension of arbitrage and its coupling with the (ir)rationality of traders (and market participants), as well as classical and quantum-like viewpoints on (ir)rationality.

Key words: Quantum-like paradigm · Arbitrage · Decision making · Risk neutral probabilities
\end{abstract}

\section{Introduction}

The concept of 'arbitrage' is of paramount importance in the theory of asset pricing. It also plays an important role in behavioral economics, since the real (or even imagined) presence of arbitrage possibilities has a powerful influence on the psychology of agents of the financial market ${ }^{1}$. In essence an arbitrage opportunity implies that a positive financial return can be realized, which is in excess of the risk free rate of interest ${ }^{2}$, on taking a trading position in an asset which entails no financial risk. Disregarding the cost which may need to

$\overline{1}$ See Shleifer \& Vishny (1997) and references on various psychological distortions in the behavior of arbitrageurs and their clients and global financial consequences of such psychological particularities.

2 The risk free rate of return is the return which is deemed to exist on financial assets which have no financial risk. 
be incurred for finding such arbitrage opportunities, one could in effect claim that an arbitrage opportunity is akin to obtaining what is often quoted in common parlance as a 'free lunch'. It is intuitive that pricing financial assets under the assumption there does not exist arbitrage, rationalizes the existence of so called 'benchmark' asset prices. As Øksendal (2004) indicates "It is not possible to make a sensible mathematical theory for a market with arbitrage" (p.26). As an example Black-Scholes option pricing theory, which commands a market of trillions of dollars, rests on the assumption of no-arbitrage ${ }^{3}$.

The conditions for no-arbitrage to occur for a discrete parameter process can be found in Harrison \& Kreps (1979). It is important to make the distinction on the required conditions for non-arbitrage, between discrete and continuous parameter processes. As mentioned in Karatzas \& Schreve (1998): "if one cannot win for certain by betting on a given process" (p.33), i.e. one can not make a riskless profit, then under a discrete parameter process most often such process will be a martingale ${ }^{4}$. However, this is not necessarily the case when a continuous parameter process is considered. Asset pricing is hinging very much so, on the concept of an equivalent martingale which in essence refers to the use of a probability which converts a semi-martingale into a martingale. Karatzas \& Schreve (1998) do mention that this type of equivalent measure "bear(s) a striking similarity to de Finetti's (1937, 1974) theory of coherent subjective probabilities and inferences..." (p. 34). We remark that in Haven \& Khrennikov (2016), we discuss subjective interpretations of probability within a quantum-like environment. From the outset, we note that since this paper will consider the notion of 'quantum-likeness', we can as well make an attempt to describe what is meant with this novel term. In Khrennikov \& Haven (2016) we mention in the preface that in fact it is the preponderance of the field of quantum information, which puts to the fore the interpretation that the wave function, a central object in quantum mechanics, is informational in nature. As we explain, the formalism of quantum mechanics can be used to describe information processing of any system, whether social or physical, with the caveat that there must be some sort of quantum feature to the system under study. We need to be careful with what we mean with "quan-

$\overline{3}$ The theory assumes a geometric Brownian motion of asset prices and such motion process invites the use of a calculus, also known under the name of 'Ito calculus'. A while ago, the mathematical finance literature considered a departure from the above Brownian motion, by considering a so called fractional Brownian motion. Such motion is characterized by a degree of memory (this refers to the so called Hurst exponent). Ito integration in that context is then replaced by a so called Wick integral (Björk \& Hult, 2005). Björk \& Hult (2005) also report on how one can (not) make arguments that such fractional Brownian motion is arbitrage free.

4 Roughly, a martingale can be defined as a conditional expectation of a variable $S_{t+1}$ (or a process of variables), where $t+1$ indicates the future given the information available up to $t, F_{t}$, such that: $E\left(S_{t+1} \mid F_{t}\right)=S_{t}$. A semi-martingale requires that either $E\left(S_{t+1} \mid F_{t}\right)>S_{t}$ or $E\left(S_{t+1} \mid F_{t}\right)<S_{t}$. 
tum feature". The utilization of wave functions, or the use of analogies with an uncertainty principle can (but does not have to) invoke quantum features. Wave functions can be found in classical mechanics. The idea of an uncertainty principle (for instance) exists in electrical engineering (via the time-frequency uncertainty principle). Where the term "quantum feature" has more traction, so to speak, is in the area where quantum probability (in decision making) is employed. In that same area do we find the difficult concept of 'context' (Khrennikov (2010)). Quantum features occur also in the use of Fisher information in economics and finance. Fisher information is narrowly linked to a particular potential function which emerges from quantum mechanics (see Hawkins and Frieden (2012).

We also note that quantum-like models describing decision making, when in particular such decision making occurs at the level of the financial market, will not match the canonical understanding of rationality (based on Savage's Bayesian approach). Quantum-like decision makers are irrational (from the viewpoint of the classical theory of rationality). At the same time they are completely rational from the quantum-like viewpoint where rational behavior corresponds to the non-Bayesian updating of probabilities, which is mathematically represented by the quantum probability calculus (Khrennikov, 2015). As was reflected in a number of publications on the theoretical analysis of arbitrage, the success and failure of arbitrageurs is closely coupled to a degree of irrationality (in the classical sense) of traders of the financial market and especially their clients (in the case arbitrageurs operate not with their own money).

Within the context of gauge field dynamics, Ilinski (2001) provides for a very elegant approach towards the formulation of arbitrage. He uses the theory of fibre bundles and the curvature of such fibre bundles to characterize the degree of arbitrage. The theory of fibre bundles can be found in Steenrod (1951) and Bishop \& Crittenden (1964). We will be very brief on this theory. However, we will be particularly interested in seeing how the concept of 'action', Ilinski (2001) introduces, can operate in a non-arbitrage context. We will therefore be keen in linking the role of action to the existence of risk neutral probabilities and state prices. Finally, a major objective of this paper will consist in characterizing arbitrage within a Fourier integral setting.

The outline of the paper is as follows. In the next section, following Ilinski (2001) we briefly introduce the notion of a fibre bundle and we attempt to show how curvature relates to the concept of arbitrage. In the section following we consider the role of linear action in the context of the (non) existence of arbitrage. We then argue how such action plays a role in risk neutral probabilities and state price formation. Section 5 of the paper introduces the idea that the risk neutral probabilities (whether they exist under the assumption arbitrage occurs or not) can also be derived from a density function generated via 
a Fourier integral. We will be careful in defining the precise (economics-based) ingredients of this Fourier integral. Sections 6 and 7 provide for two applications. Those two applications also go into quite some detail in showing how interaction, arbitrage and the Fourier integral may be linked together. Section 8 summarizes the two applications and we conclude the paper in section 9 .

\section{Fibre bundles and curvature}

\subsection{A short introduction}

Fibre bundles are in fact quite easy objects to define in plain language. Since this paper does not consider fibre bundles as the central object of study, we will introduce the concepts in an as simple way as possible. Ilinski (2001) says that "each fibre bundle consists of identical subspaces that are all collected together... to give the whole space" (p. 19). Ilinski (2001, p.21) shows a simple example of a tube which can be seen as a fibre bundle with a circular base and line fibres. A more involved example may consist of a fibre bundle which is a "spherical base and the fibres which are two dimensional tangent planes stuck to each point of the sphere." (Ilinski, 2001, p. 25). We note that the fibre bundle is known under other names such as: 'fibration'; 'twisted product' or also 'Steenrod bundle' (see Monastyrsky, 1993, p.48). Thus, we can intuitively grasp (in a very informal way) that a fibre bundle can be a manifold with a base which is a manifold. Fibres, $F$, which make up the fibre bundle are also

manifolds. Now, one can imagine a rule for the 'movement' of an element of the fibre, say $x$, from one point of the base to another point of the base, say $y$. An operator, $U(\gamma)$ can be defined which describes a 'movement' along some curve $\gamma ; U(\gamma): F_{x} \rightarrow F_{y}$. See below for more details (especially Definition 5).

\subsection{Fibre bundle operators and curvature}

Ilinski (2001) was the first to introduce and fine tune the use of fibre bundles in an economics context. The objective of this subsection is to briefly describe a simple example where the curvature idea can be easily explained. Examples 1 and 2; Claims 3 and 4 and Definitions 5 and 6; and Example 7 below follow Ilinski, 2001, p. 88-90.

Example 1 Assume we have two assets ( $i$ and $i+1)$ : cash and a share of a stock of some company. Then define $U((i, n),(i, n+1))=e^{r_{1} \Delta}$; where $r_{1}$ is the return on a share of a company and $\Delta$ is the equally spaced time difference (between times $n+1$ and $n$ ). Hence, if one has 1 unit of currency to invest 
in a company share over a period of time $\Delta$ then one obtains (continuously compounded): $e^{r_{1} \Delta}$. Now define $U((i+1, n),(i+1, n+1))=e^{r_{0} \Delta}$, where $r_{0}$ is the return on cash and $\Delta$ is the equally spaced time difference. The inverse movement can be defined too: $U((i+1, n+1),(i+1, n))=e^{-r_{0} \Delta}$ (this is the present value of 1 unit of currency). Similarly for the inverse movement, relative to $U((i, n),(i, n+1))$, which is $U((i, n+1),(i, n))=e^{-r_{1} \Delta}$. Now consider $U((i, n),(i+1, n))=S_{i}$, where $S_{i}$ is the price in unit of currency for the share of a company (buy a share). One share is thus exchanged on $S_{i}$ units of cash at some point in time $t_{i}$. Define now also $U((i, n+1),(i+1, n+$ $1))=S_{i+1}$, where $S_{i+1}$ is the price in unit of currency for the share of a company (buy a share) at some point in time $t_{i+1}$. The inverse movement can be defined too: $U((i+1, n),(i, n))=S_{i}^{-1}$ (sell share at time $\left.t_{i}\right)$. Similarly for the inverse movement: $U((i+1, n+1),(i, n+1))=S_{i+1}^{-1}$ (sell share at time $\left.t_{i+1}\right)$.

A very interesting question is the following: how can an investor who has cash at time $t_{i}$ buy shares at time $t_{i+1}$ ? There are two straightforward ways to do so. Let us consider another example to illustrate this.

Example 2 One can put cash in the bank and get $r_{0}$ return at time $t_{i+1}$ and then withdraw money at $t_{i+1}$ and buy shares $S_{i+1}$. The investor gets the amount of shares: $\frac{e^{r_{0} \Delta}}{S_{i+1}}$, which is the amount of money divided by the price of the share at $t_{i+1}$. In other words, $\frac{e^{r_{0} \Delta}}{S_{i+1}}$ is the amount of shares at $t_{i+1}$ for each unit of cash invested at time $t_{i}$. Another way to get shares at time $t_{i+1}$ is to buy shares at price $S_{i}$ at time $t_{i}$. In other words, $\frac{e^{r_{1} \Delta}}{S_{i}}$ is the amount of shares at $t_{i+1}$ for each unit of cash invested at time $t_{i}$.

For the purposes of defining curvature (see Definition 6 below) in an economics context, it is important to query what happens when $\frac{e^{r_{0} \Delta}}{S_{i+1}} \neq \frac{e^{r_{1} \Delta}}{S_{i}}$ ? In that case a possible arbitrage opportunity looms! Consider the following two claims.

Claim 3 If $\frac{e^{r_{0} \Delta}}{S_{i+1}}<\frac{e^{r_{1} \Delta}}{S_{i}} \Leftrightarrow \frac{e^{r_{1} \Delta}}{S_{i}} \frac{S_{i+1}}{e^{r_{0} \Delta}}>1$ which is equivalent to an arbitrage

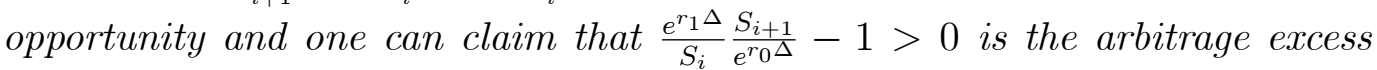
return.

Claim 4 If $\frac{e^{r_{0} \Delta}}{S_{i+1}}>\frac{e^{r_{1} \Delta}}{S_{i}} \Leftrightarrow \frac{e^{r_{0} \Delta}}{S_{i+1}} \frac{S_{i}}{e^{r_{1} \Delta}}-1>0$ there is again another arbitrage excess return.

Definition 5 The transactions exemplified above can then be more generally defined as $U(\gamma)=\prod_{i=1}^{p-1} U\left(x_{i}, x_{i+1}\right)$; where $U\left(x_{i}, x_{i+1}\right) \in G$, and $G$ is called a structure group (of transformations of $F$ ). Recall how the above short introduction (section 2.1.) was concluded with the definition of an operator, $U(\gamma): F_{x} \rightarrow F_{y}$. The curve $\gamma$ can be defined as: $\gamma \equiv\left\{x_{i}\right\}_{i=1}^{p-1}$. 
Definition 6 The arbitrage excess return as put forward in Claims 3 and 4 is then defined as: $R=\prod_{i=1}^{p-1} U\left(x_{i}, x_{i+1}\right)-1$; and $R$ is an indicator of curvature.

Example 7 Using Claims 3 and 4 , one can slightly generalize $R=\prod_{i=1}^{p-1} U\left(x_{i}, x_{i+1}\right)-$ 1 as in Definition 6 above, to write immediately that: $R^{\prime}=e^{r_{1} \Delta} S_{i}^{-1} S_{i+1} e^{-r_{0} \Delta}+$ $e^{r_{0} \Delta} S_{i+1}^{-1} S_{i} e^{-r_{1} \Delta}-2$. Here, $R^{\prime}$ is also an indicator of curvature.

To highlight a little better the idea of the curvature, let us consider the following figure.

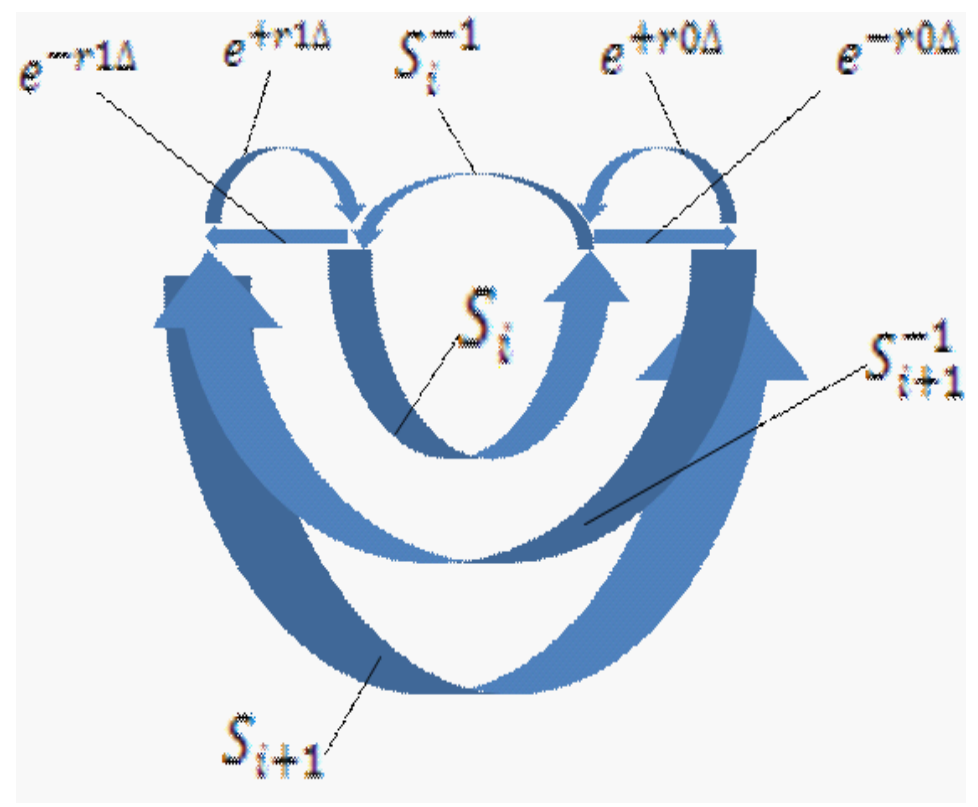

Fig. 1. Torus shape rendering of $R^{\prime}$

The arrow length in figure 1 could be seen as an indicator of the values of the various components in $R^{\prime}$. As an example $S_{i}>S_{i}^{-1}$ as one would expect for $S_{i}>1$. We could propose that the figure above could be seen as one of the equivalent manifolds of a torus, overlaid on a sphere. The so called 'Gaussian curvature' of a torus can be seen to be zero ${ }^{5}$. This does not mean that no measure of arbitrage can be calculated. Indeed, the measure $R^{\prime}$ in $R^{\prime}=$ $e^{r_{1} \Delta} S_{i}^{-1} S_{i+1} e^{-r_{0} \Delta}+e^{r_{0} \Delta} S_{i+1}^{-1} S_{i} e^{-r_{1} \Delta}-2$ shows that when there is arbitrage it must be that $e^{r_{1} \Delta} S_{i}^{-1} S_{i+1} e^{-r_{0} \Delta}+e^{r_{0} \Delta} S_{i+1}^{-1} S_{i} e^{-r_{1} \Delta}>2$, and therefore $R^{\prime}>0$.

$\overline{5}$ Ilinski (2001, p. 59) shows that by using the so called Euler characteristic of the manifold (which is 0 in the case of the torus), one can calculate via the so called Gauss-Bonnet theorem, the curvature. 


\section{The concept of action in an arbitrage setting}

The beauty of Ilinski's work not only resides in the way he connects the idea of curvature with arbitrage but also in a precious additional insight his theory gives: to connect curvature with the concept of 'action'

Definition 8 Ilinski (2001, p.95). An action which is linear in the curvatures, $R$, is defined $a^{6}: \mathcal{A}(\{S, r\})=\sum_{\gamma} \alpha_{\gamma}\left(1+R_{\gamma}\right)$; where $\gamma$ is a curve (see Definition 5); $\alpha_{\gamma}$ is some positive coefficient and $R_{\gamma}$ is curvature.

Ilinski (2001, p. 96) shows that by using $R^{\prime}=e^{r_{1} \Delta} S_{i}^{-1} S_{i+1} e^{-r_{0} \Delta}+e^{r_{0} \Delta} S_{i+1}^{-1} S_{i} e^{-r_{1} \Delta}-$ 2 (Example 7), the action becomes:

$$
\mathcal{A}(\{S, r\})=\sum_{i=-\infty}^{\infty} \alpha_{i}\left(e^{r_{1} \Delta} S_{i}^{-1} S_{i+1} e^{-r_{0} \Delta}+e^{r_{0} \Delta} S_{i+1}^{-1} S_{i} e^{-r_{1} \Delta}-2\right) .
$$

We can thus claim that under no arbitrage, for any stock price, $S_{i}: \sum_{i=-\infty}^{\infty} \alpha_{i} \cdot(0)=$ 0 . Thus, $\mathcal{A}(\{S, r\})=0$ in the case there does not exist any arbitrage.

Ilinski (2001, p. 96) shows that (1) can be re-written in a different way. Under the choice of letting the time step $\Delta \rightarrow 0$ and setting $\alpha_{i}=\frac{1}{2 \Delta \sigma_{i}^{2}}$, he writes:

$$
\mathcal{A}(\{S, r\})=\frac{1}{2} \int_{-\infty}^{\infty} \frac{1}{\sigma^{2}(t)}\left(\frac{d S}{d t} \frac{1}{S}-\mu\right)^{2} d t
$$

which, as Ilinski (2001) indicates, corresponds to a geometric random walk (with time dependent volatility) and $\mu$ is seen here as an average return. We note that the above result was already found in 1997 by Belal Baaquie (1997).

Claim 9 From (1) it can be seen that no-arbitrage in (2), generates the implication: $\frac{d S}{d t} \frac{1}{S}=\mu \Rightarrow \mathcal{A}(\{S, r\})=0$; where $d S=\mu S d t$ thus implies a zero action.

From the above claim it can also be seen that if $\mathcal{A}(\{S, r\}) \neq 0$ then there exists arbitrage. The existence of arbitrage means that $\frac{d S}{d t} \frac{1}{S}>\mu$ or $\frac{d S}{d t} \frac{1}{S}<\mu$. Some observations are in order now.

- The equality ensuing from the no arbitrage condition, $d S=\mu S d t$, implies the absence of a stochastic price process generator, which we know from (2) was geometric Brownian motion. Thus, when $\mathcal{A}(\{S, r\}) \neq 0$ with the linear action as in Definition 8, we have $d S=\mu S d t+\sigma S d W$, where $d W$ is

${ }_{6}$ Quadratic action can also be introduced. See Ilinski (2001, p. 98). Those are very rich functionals which we however do not use in this paper. 
the Wiener process and $\sigma$ is the volatility. This is intuitive since indeed the existence of arbitrage means that $d S>\mu S d t$ or $d S<\mu S d t$. We assume in the sequel of the paper that the volatility is time independent.

- In Claim 9, the return per unit of time is equal to the return, $\mu$. But since the absence of arbitrage implies $\mathcal{A}(\{S, r\})=0$ and since there is no stochastic process, we could claim that this return should be the risk free rate of return. I.e. with the existence of a stochastic process the use of a risk free rate would not be possible. We assume at a given instant of time there exists only one risk free rate of interest, $r_{f}$.

- We note the ideas of least action can in some sense be applied here. Considering either (1) or (2), we can wonder which result of the financial transaction leads to the lowest value of action. This very transaction can then be denoted as the 'least action' transaction. If nil is our minimum, then the no-arbitrage transaction would be the least action.

\section{Risk neutral probabilities and action}

Ilinski (2001, p. 93-94) defines the probability distribution of values of $U\left(x_{i}, x_{i+1}\right)$. We recall that $U\left(x_{i}, x_{i+1}\right)$ can be recognized in Definitions 5 and 6 . If we assume that interest rates are given, then this probability distribution in effect does refer to the values of the shares, i.e. the stock prices.

Definition 10 Ilinski (2001, p. 93-94). $P(\{S, r\})=N \exp (-\mathcal{A}(\{S, r\}))$; where $N$ is a normalization factor.

We note that the normalization factor $N$ is not a free parameter ${ }^{7}$

Our understanding is that action contains two essential variables: time and position. We can write:

$$
\mathcal{K}\left(S_{a}, S_{b}, t_{i}, t_{f}\right)=N \Pi_{t=t_{i}}^{+\infty} \int_{t=t_{i}}^{t=t_{f}} d x(t) \exp \left(-\mathcal{A}\left(S_{a}, S_{b}, t_{i}, t_{f}\right)\right)
$$

where $\mathcal{A}$ is action; $S_{a}, S_{b}$ indicate asset positions at respectively, time $t_{i}$ and time $t_{f}$; where $t_{i}<t_{f}$. See Baaquie (2004). Note the product operator whose role it is to multiply amplitudes to go, say from say $x_{1}$ to $x$ over time interval $t / 2$ and from $x$ to $x_{2}$ over time interval $t / 2$. Intuitively, the multiplication of such amplitudes refers back to the intersection of events. A key argument in path integration is that paths are divided up to signal the presence of the uncertainty relation in quantum mechanics.

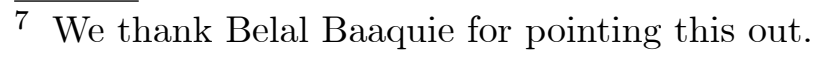


Baaquie (2004) has shown that the conditional probability for observing $S_{b}$ at time $t_{f}$ given it was first observed at position $S_{a}$ at time $t_{i}$ is:

$$
\mathcal{K}\left(S_{a}, S_{b}, t_{i}, t_{f}\right)=N \exp \left(-\frac{1}{2 \int_{t_{i}}^{t_{f}} d t \sigma^{2}(t)}\left(S_{b}-S_{a}-\left(t_{f}-t_{i}\right) \mu\right)^{2}\right)
$$

where $\sigma^{2}(t)$ time dependent stock price volatility.

The following question may emerge. Is the conditional probability $p\left(S_{b}, t_{f} \mid S_{a}, t_{i}\right)$ (that $S_{b}$ is observed at $t_{f}$ given it was at position $S_{a}$ at time $t_{i}$ ), comparable to the (conditional) probability in the non-arbitrage theorem, where each risk neutral probability could be seen as a conditional probability given a state of nature occurring?

Are those types of probabilities intimately related to the so called fundamental theorem of asset pricing (Harrison and Kreps , 1979)? In this paper, we follow the formulation of the theorem as in Etheridge (2002). See also Haven (2008, p.194), where we cite that theorem also:

Theorem 11 Assume there are $N$ tradable assets (some assets may be risky and some not) and their prices, at time $t_{0}$ are given by $\overrightarrow{p_{0}}=\left(p_{0}^{1}, p_{0}^{2}, . . p_{0}^{N}\right)$. Assume there exists a $K$ (where $K$ indicates the $K$ states of the world) dimensional state price vector $\vec{\Phi}=\left(\Phi_{1}, \Phi_{2}, . . \Phi_{K}\right)$ which is strictly positive in all coordinates. Consider the following model: $\left(\begin{array}{c}p_{0}^{1} \\ p_{0}^{2} \\ \cdot \\ \cdot \\ p_{0}^{N}\end{array}\right)=\Phi_{1}\left(\begin{array}{c}D_{11} \\ D_{21} \\ \cdot \\ \cdot \\ D_{N 1}\end{array}\right)+\Phi_{2}\left(\begin{array}{c}D_{12} \\ D_{22} \\ \cdot \\ \cdot \\ D_{N 2}\end{array}\right)+$ ..$\Phi_{K}\left(\begin{array}{c}D_{1 K} \\ D_{2 K} \\ \cdot \\ \cdot \\ D_{N K}\end{array}\right)$, where each $N$ dimensional vector $\overrightarrow{D_{1}}, . . \overrightarrow{D_{K}}$ is the security price vector at time $t_{1}$, if the market is, respectively, in state $1, . . K$. For the market model described here there is no arbitrage if and only if there is a state price vector.

The so called risk neutral probabilities are given by the vector, $\overrightarrow{\Phi_{\text {prob }}}=$ $\left(\frac{\Phi_{1}}{\Phi_{0}}, \frac{\Phi_{2}}{\Phi_{0}}, \frac{\Phi_{3}}{\Phi_{0}}, \ldots \frac{\Phi_{K}}{\Phi_{0}}\right)$, where each coordinate is a probability and $\Phi_{0}=\exp \left(-r_{f} T\right)$ 
is the discount rate (continuously discounted) at the risk-free rate of return, $r_{f}$, and $T$ is time. As is stated in Etheridge $(2002), E^{\overrightarrow{\Phi_{\text {prob }}}}\left(p_{T}^{j}\right)=\sum_{i=1}^{K} D_{i j} \frac{\Phi_{i}}{\Phi_{0}}$, where $i=1, \ldots K$ and $j=1, \ldots N$ and where $E^{\overrightarrow{\Phi_{p r o b}}}$ is the expectation operator under the risk-neutral probability measure. We note that the probabilities are duly normalized by imposing that $p_{0}^{1}=1$ and by further imposing that $D_{11}=D_{12}=\ldots D_{1 K}=\Phi_{0}$. This normalization procedure is well known in financial economics and can be found back in Neftci (2000).

We could write the risk neutral probabilities in the non-arbitrage theorem as: $p\left(. \mid\right.$ state price, $\left.t_{1}\right)$, since the risk neutral probability (for 2 states of the world for instance) is defined as: $p_{1}=\left(1+r_{f}\right) \Phi_{1}$, thus $p_{1}$ is conditional upon the state price $\Phi_{1}$ and it occurs in the future at time $t_{1}$. In effect, like in binomial option pricing, the probability, $p$ is the probability of the price to be $S . u$, with $u>1$, in the next instant; given it is $S$ now. The complement probability, $1-p$, is then the probability of the price to be $S . d$, with $d<1$, in the next instant; give it is $S$ now. In analogy with the binomial option pricing model, we could write: $p\left(S . u, t_{1} \mid S, t_{0}\right)$ and $p\left(S . d, t_{1} \mid S, t_{0}\right)=1-p\left(S . u, t_{1} \mid S, t_{0}\right)$. So similarly within the non-arbitrage theorem, we could write: $p\left(S_{1}, t_{1} \mid S_{0}, t_{0}\right)$ and $p\left(S_{2}, t_{1} \mid S_{0}, t_{0}\right)$. Hence, we would like to argue that in that sense will those probabilities be similar to the conditional probability: $p\left(S_{b}, t_{f} \mid S_{a}, t_{i}\right)$.

We also note that $\mathcal{K}\left(S_{a}, S_{b}, t_{i}, t_{f}\right)=N \exp \left(-\frac{1}{2 \int_{t_{i}}^{t_{f}} d t \sigma^{2}(t)}\left(S_{b}-S_{a}-\left(t_{f}-t_{i}\right) \mu\right)^{2}\right)$ as found in Baaquie (2004) is not the result of non-arbitrage. Consider: $\mathcal{K}\left(S_{a}, S_{b}, t_{i}, t_{f}\right)=$ $N \Pi_{t=t_{i}}^{t_{f}} \int_{t=t_{i}}^{t=t_{f}} d x(t) \exp \left(-\mathcal{A}\left(S, r, t_{i}, t_{f}\right)\right)$, and assume it contains action $\mathcal{A}(\{S, r\})=$ $\frac{1}{2} \int_{-\infty}^{\infty} \frac{1}{\sigma^{2}(t)}\left(\frac{d S}{d t} \frac{1}{S}-\mu\right)^{2} d t$

However, as we said above, in claim 9: $\frac{d S}{d t} \frac{1}{S}=\mu \Rightarrow \mathcal{A}(\{S, r\})=0$. Thus, under non-arbitrage what happens then to: $\mathcal{K}\left(S_{a}, S_{b}, t_{i}, t_{f}\right)=N \Pi_{t=t_{i}}^{+\infty} \int_{t=t_{i}}^{t=t_{f}} d x(t) \exp \left(-\mathcal{A}\left(S, r, t_{i}, t_{f}\right)\right)$ when $\mathcal{A}(\{S, r\})=0$ ? We can find that:

$N \Pi_{t=t_{i}}^{+\infty} \int_{t=t_{i}}^{t=t_{f}} d x(t)=N \Pi_{t=t_{i}}^{+\infty}[x(t)]_{t_{i}}^{t_{f}}=N \Pi_{t=t_{i}}^{+\infty}\left(x_{t_{f}}-x_{t_{i}}\right)=N\left[\begin{array}{c}\left(x_{t_{f}}-x_{t_{f-1}}\right)\left(x_{t_{f-1}}-x_{t_{f-2}}\right) \\ \left(x_{t_{f-2}}-x_{t_{f-3}}\right) \\ \left(x_{t_{f-3}}-x_{t_{f-4}}\right) \ldots . .\left(x_{t_{f-n}}-x_{t_{0}}\right)\end{array}\right]$. 
4.1 Risk neutral and binomial option pricing probabilities and the normalization factor

We proceed in this paper with the following Claim.

Claim 12 The probability values which are part of $\overrightarrow{\Phi_{\text {prob }}}$ can be matched to the probabilities obtained in $\mathcal{K}\left(S_{a}, S_{b}, t_{i}, t_{f}\right)$ when $\mathcal{A}\left(S, r, t_{i}, t_{f}\right)=0$.

Definition 13 The risk neutral probability in the binomial option pricing model is (under no-arbitrage conditions) $:$ prob bin $_{b}=\frac{\exp \left(r_{f} T\right)-d}{u-d}$, where $d$ is the down proportion for the underlying asset price (like a stock price) to go down and $u$ is the up proportion for the underlying asset price to go up.

Let us consider a simple case of $K=2$ states of the world with $N=2$ assets. Using the theorem above we then can simply write, using $T=1$ and using instead of $\exp \left(r_{f}\right), 1+r_{f}: p_{0}^{j}=\frac{1}{1+r_{f}}\left[D_{j 1} \frac{\phi_{1}}{\phi_{0}}+D_{j 2} \frac{\phi_{2}}{\phi_{0}}\right]$. Therefore, we can write that: $E^{\overrightarrow{\Phi_{\text {prob }}}}\left(p_{1}^{j}\right)=\left(1+r_{f}\right) p_{0}^{j}$. We obtain an identical result when we use the probability value used in the binomial option pricing model: $E^{\text {prob }}$ $\frac{1+r_{f}-d}{u-d} p_{0}^{j} u+\left(1-\frac{1+r_{f}-d}{u-d}\right) p_{0}^{j} d=\left(1+r_{f}\right) p_{0}^{j}$. Clearly, in this very case, $D_{j 1}=p_{0}^{j} u$ and $D_{j 2}=p_{0}^{j} d$. We could thus set: prob $_{b i n}=\frac{\phi_{1}}{\phi_{0}}$. From Claim 12, imposing that prob $_{b i n}=$ prob, what do we find?

What we do with the path integral approach is to divide up a path. Now in a one step binomial tree you just have simply $S->S . u$ or $S->S . d$. The amplitude is an integral over all possible paths between the end points. The particle does not travel along a particular path. Imagine a multistep binomial tree, with very small time steps. We could follow one path, when the price goes up only. Or another path: when the price goes up first and then down etc...Thus, a multistep binomial tree clearly draws many possible paths. Each time step is characterized by a probability to go up or down (in some sense a conditional probability). The next issue becomes as follows. Trading is not continuous. Hence, there must be 'holes' in the price path. It is precisely the presence of the holes which mimics the existence of an uncertainty principle. In some sense, without reaching the continuous limit, the binomial model does just that: it has holes. Hence, we could set:

$$
\frac{1+r_{f}-d}{u-d}=N\left[\begin{array}{c}
\left(x_{t_{f}}-x_{t_{f-1}}\right)\left(x_{t_{f-1}}-x_{t_{f-2}}\right)\left(x_{t_{f-2}}-x_{t_{f-3}}\right) \\
\left(x_{t_{f-3}}-x_{t_{f-4}}\right) \ldots . .\left(x_{t_{f-n}}-x_{t_{0}}\right)
\end{array}\right] .
$$

We must be careful though to realize that $N$, the normalization factor is NOT a free parameter. We now have the following claim: 
Claim 14 If the state price vector $\vec{\Phi}^{\prime}=\left(\Phi_{1}^{\prime}, \Phi_{2}^{\prime}, . . \Phi_{K}^{\prime}\right)$ does not solve the market model as described in theorem 11 above, we claim that $\overrightarrow{\Phi_{\text {prob }}}=\left(\frac{\Phi_{1}^{\prime}}{\Phi_{0}}, \frac{\Phi_{2}^{\prime}}{\Phi_{0}}, \frac{\Phi_{3}^{\prime}}{\Phi_{0}}, \ldots \frac{\Phi_{K}^{\prime}}{\Phi_{0}}\right)$ can be drawn from $\mathcal{K}\left(S_{a}, S_{b}, t_{i}, t_{f}\right)=N \Pi_{t=t_{i}}^{+\infty} \int_{t=t_{i}}^{t=t_{f}} d x(t) \exp \left(-\mathcal{A}\left(S, r, t_{i}, t_{f}\right)\right)$ when $\mathcal{A}(\{S, r\}) \neq 0$ (i.e. there is arbitrage).

\section{A quantum-like environment: Fourier transforms, arbitrage and risk neutral probabilities}

\subsection{Introduction}

In this section of the paper we attempt to show how the Fourier integral can be used to benefit in the arbitrage environment we have described above. The development in this section will also lead to the introduction of related ideas, which we hope can be of interest to further characterize the phenomenon of arbitrage in a quantum-like environment.

Definition 15 Kreyszig (1999, p. 570). A Fourier transform of a function $f$ can be defined as $: \hat{f}(w)=\frac{1}{\sqrt{2 \pi}} \int_{-\infty}^{\infty} f(x) \exp (-i w x) d x$; where $i$ is an imaginary unit. Note that $f(x)$ i) needs to be piecewise continuous on every finite interval and ii) $f(x)$ is absolutely integrable on the $X$-axis

Definition 16 Kreyszig (1999, p. 570). The Fourier integral is defined as: $f(x)=\frac{1}{\sqrt{2 \pi}} \int_{-\infty}^{\infty} \widehat{f}(w) \exp (i w x) d w$.

In the introduction of this paper, we already mentioned the 'quantum-like' paradigm to denote essentially the use of elements of the formalism of quantum mechanics in areas outside of physics. Central to this paradigm is the notion that the wave function, the central object in quantum mechanics, has an information based character. The quantum-like paradigm has now been the main topic of several monographs and many papers (see for example, Basieva et al., 2010; Khrennikov, 2010, 2015; Haven \& Khrennikov, 2013; Piotrowski \& Sładkowski, 2003, 2004; Asano et al. , 2011, 2015; Busemeyer \& Bruza, 2012; Plotnitsky, 2009, 2014; Dzhafarov et al., 2015; Dzhafarov \& Kujala, 2012, 2014; Baaquie, 2004, 2013, 2014; Yukalov \& Sornette, 2011, 2014; Hawkins \& Frieden, 2004, 2012; Frieden \& Hawkins, 2010; Hawkins et al., 2010; Aerts et al., 2013 and other work). A key paper which started, to a large extend, the quantum-like paradigm is by Khrennikov (1999).

Although the application of wave functions, when not sourced from quantum mechanics, have also found uses in social science, especially economics (see for example, Haven et al., 2009), our paper thus looks at wave functions as formalized via Fourier transforms and integrals which are intimately intertwined 
with the existence of the Heisenberg uncertainty principle. It is now our intention to introduce the 'wave number - density function' device which forms the cornerstone of basic quantum mechanics, in the context of arbitrage. We believe that the use of such device may provide for some interesting insights. In building up our argument, we will also actively refer back to the role the action functional $\mathcal{A}(\{S, r\})$ plays in arbitrage (see (2) and Claim 9).

\subsection{The wave number, volatility and an uncertainty principle}

The Fourier transform and integrals we defined above form the backbone of basic quantum mechanics. However, those mathematical instruments are also widely used outside of quantum mechanics.

The wave number, often denoted as $k$, is the input in the so called amplitude function, $A(k)$ and it plays an important role in the wave function, which we will denote here as $\Psi(S, t)$, where $S$ denotes position and $t$ time. Remark that the variable ' $w$ ' is the wave number in Definition 16 . The wave function plays an important role in the determination of the probability density function (pdf) which measures the likelihood of a position, $S$. Such pdf is obtained by multiplying the wave function with its conjugate. Those concepts will be put in an economics setting in this (and the next) subsections.

It is probably reasonable to expect that any keen reader of this paper, after having embarked on reading section 5, will now have a set of pressing questions to ask. We can imagine questions like: "What is the point of introducing those very basic quantum mechanical concepts in an economics context?" or also "What problem is being solved?" or also "Which added clarity is yielded by using those very exotic concepts?" We hope to answer those essential questions in what follows. Let us quickly consider the Heisenberg uncertainty principle. We could possibly translate this uncertainty principle into an economics context by naively writing that ${ }^{8}$ :

$$
\Delta S \Delta p \geq \frac{\sigma^{2}}{2}
$$

where $\Delta S$ and $\Delta p$ indicate the imprecision on position and momentum, respectively. The squared volatility, $\sigma^{2}$, can be found back in the commutation equation Baaquie (2004, p. 99), presents.

Definition 17 Baaquie (2004, p. 99). The commutation equation is defined as: $E\left[S_{n}\left(\frac{S_{n+1}-S_{n}}{\tau}\right)-\left(\frac{S_{n}-S_{n-1}}{\tau}\right) S_{n}\right] \equiv \sigma^{2}$; where $\tau$ is time, and $n(n \pm 1)$ indicates the value of $S$ at present (future/past).

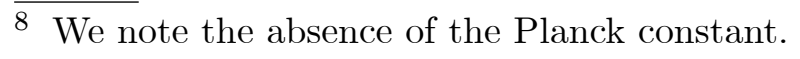


We note that this same volatility is also to be found back in geometric Brownian motion (see section 3 of the paper): $d S=\mu S d t+\sigma S d W$.

We can re-write Definition 17 by letting $p=\frac{S_{n+1}-S_{n}}{\tau}$ and $p^{\prime}=\frac{S_{n}-S_{n-1}}{\tau}$, as $E\left(S_{n}\left(p-p^{\prime}\right)\right)$. If $\Delta p=p-p^{\prime}$, measures the uncertainty on momentum, then it must be that $\Delta p=0$ if there exists a precise momentum.

It can be of possible interest to observe what the economics meaning is in Definition 17 from having $p=p^{\prime}$. Here is a very simple result.

Proposition 18 If no uncertainty on momentum exist then by using Definition 17, we have that $E(R)=\frac{S_{n-1}}{S_{n}} R^{\prime}$, where $E(R)$ is the expected return given the future asset price and $R^{\prime}$ is the return given the past asset price.

PROOF. The proof is obvious. Using Definition 17 and setting $\Delta p=p-p^{\prime}=$ 0 , it is immediate that $S_{n}$ is the average of the future asset price, $S_{n+1}$, and the past asset price, $S_{n-1}$. Hence, we can write: $S_{n+1}-S_{n}=S_{n}-S_{n-1}$ and then dividing by $S_{n} S_{n-1}: \frac{S_{n+1}-S_{n}}{S_{n} S_{n-1}}=\frac{S_{n}-S_{n-1}}{S_{n} S_{n-1}}$, we get: $\frac{1}{S_{n-1}} R=\frac{1}{S_{n}} R^{\prime}$, where $R$ is the return on the future asset and $R^{\prime}$ is the return on the past asset. We can then write: $E(R)=\frac{S_{n-1}}{S_{n}} R^{\prime}$, which indicates that the expected return on the future asset price is known with certainty to be equal to the past return multiplied with a factor of the ratio of past over current price.

Thus, we have clearly a unique return (i.e. really a known number) when $\Delta p=0$, which is indeed what we wanted - i.e. there is no uncertainty on the momentum. We can define the momentum in a slightly more refined way:

Definition 19 The momentum $p$ can be defined in an economics context as: $p=\frac{d S}{d t}$, i.e. the first derivative of position towards time.

The wave number, $k$, which we mentioned at the beginning of this section, can be defined as:

Definition 20 The wave number, $k$ can be defined in an economics context as: $k=\frac{p}{\sigma^{2}}$, where $\sigma^{2}$ is the squared volatility we mentioned at the beginning of this section.

Give the above two definitions, the wave number, $k$, can then be written as:

$$
k=\frac{1}{\sigma^{2}} \frac{d S}{d t} .
$$

The Fourier integral, which entirely copies Definition 16 is now simply: 


$$
\Psi(S)=\frac{1}{\sqrt{2 \pi}} \int_{-\infty}^{\infty} A(k) \exp (i k x) d k
$$

where we have assumed a time independent wave function.

\subsection{No arbitrage, uniqueness of the wave number and the uncertainty prin- ciple}

Let us now revisit the no arbitrage condition contained in Claim 9. The condition, $\frac{d S}{d t} \frac{1}{S}=r_{f}$ for the action functional $\mathcal{A}(\{S, r\})=0$, can be slightly re-written as: $\frac{d \log S}{d t}=\frac{d S^{\prime}}{d t}$ and therefore, using $k=\frac{1}{\sigma^{2}} \frac{d S}{d t}$, we can then write the no-arbitrage condition, Claim 9 , with the wave number as:

$$
k=\frac{1}{\sigma^{2}} r_{f} \Rightarrow \mathcal{A}(\{S, r\})=0
$$

Using the naive uncertainty principle mentioned in (7), the above implication (10), would then indicate that there does not exist any uncertainty on $k$, i.e. $\Delta k=0$. Since, $k=\frac{p}{\sigma^{2}}$ then $\Delta k=\frac{\Delta p}{\sigma^{2}}$. Thus, we can write:

$$
k=\frac{1}{\sigma^{2}} r_{f} \Rightarrow \Delta p=0 \Rightarrow \mathcal{A}(\{S, r\})=0
$$

If we now want to use (9), given the existence of no uncertainty on the wave number, $k$, then the amplitude function $A(k)$, would need to be of the DiracDelta type. This would imply that the uncertainty on position, $S$, would have to be infinitely wide, i.e. $\Delta S \rightarrow \infty$. The pdf derived from (9) gives the pdf on the position $S$, and it can be derived by multiplying the wave function with its conjugate. This will be discussed further in section 7 of this paper. But see also section 5.5. for a simple example.

The volatility in $\Delta S \Delta p \geq \frac{\sigma^{2}}{2}$ can not be zero as else i) the wave number becomes infinite and ii) the action functional $\mathcal{A}(\{S, r\})=\frac{1}{2} \int_{-\infty}^{\infty} \frac{1}{\sigma^{2}(t)}\left(\frac{\partial S}{\partial t} \frac{1}{S}-\mu\right)^{2} d t$ will obviously be ill-defined.

We would like to argue that the presence of arbitrage, will make $\frac{\partial S}{\partial t} \frac{1}{S} \neq r_{f}$. The non-equality to the risk free rate of the return on $S$ per unit of time, could imply that the return is now un-predictable. We could invoke a stochastic driver process such as the Wiener process. This Wiener process can be approximated in discrete time format as: $d W \sim \epsilon \sqrt{\Delta t}$, where $\epsilon$ follows a Normal density with mean 0 and variance 1 , i.e. $N(0,1)$. Since $\mathcal{A}(\{S, r\})=$ 
$\frac{1}{2} \int_{-\infty}^{\infty} \frac{1}{\sigma^{2}(t)}\left(\frac{\partial S}{\partial t} \frac{1}{S}-\mu\right)^{2} d t$ corresponds to a geometric Brownian motion, we can have as a stochastic driver, in discrete time:

$$
\sigma S d W \sim \sigma S \epsilon \sqrt{\Delta t}
$$

The absence of arbitrage may require either $\sigma=0$ or $\epsilon=0$. As discussed above, setting $\sigma=0$ leads to ill defined wave numbers and action functionals. Hence, we make the following claim.

Claim 21 We claim the uncertainty principle in an economics context to be defined as $\Delta S \Delta p \geq \frac{\sigma^{2}}{2} \epsilon$, where $\epsilon \sim N(0,1)$.

Following (10), in a non-arbitrage situation, $\Delta p=0$ (i.e. we have a precise momentum) and since we can not have that $\sigma=0$, we impose instead that $\epsilon=0$. This is reasonable. After all, following Claim 9, no-arbitrage means that the action functional $\mathcal{A}(\{S, r\})=\frac{1}{2} \int_{-\infty}^{\infty} \frac{1}{\sigma^{2}(t)}\left(\frac{\partial S}{\partial t} \frac{1}{S}-\mu\right)^{2} d t=0$ and this implies the absence of a geometric Brownian motion, and thus the absence of a stochastic driver. In other words, $\epsilon=0$ in $\sigma S \epsilon \sqrt{\Delta t}$ is reasonable. Claim 21, allows the wave number to still be defined and the action functional too.

However, the problem of certainty in position or momentum is in fact not solved. It can be shown that a precise momentum, via the use of the Fourier transform, even though we were to use the altered uncertainty relation $\Delta S \Delta p \geq$ $\frac{\sigma^{2}}{2} \epsilon$, will still yield an infinite uncertainty on position. This then certainly means that in the non-arbitrage case, we can not use the wave function as an input to construct a density function from which we could source potential risk neutral probabilities.

\subsection{Arbitrage, non-uniqueness of the wave number and the uncertainty prin- ciple}

In the case of the existence of arbitrage, we obtain the existence of the Wiener process. Let us write, $\frac{\Delta S}{\Delta t} \frac{1}{S}=\frac{\Delta \log S}{\Delta t}$. With arbitrage, we write: $\frac{\Delta S}{\Delta t} \frac{1}{S}=\mu+$ $\sigma \epsilon \frac{1}{\sqrt{\Delta t}}$ and therefore: $\frac{\Delta \log S}{\Delta t}=\mu+\sigma \epsilon \frac{1}{\sqrt{\Delta t}}$. Note that $\mu$ is not the risk free rate of return.

Using (8), let us write $k=\frac{1}{\sigma^{2}} \frac{\Delta S^{\prime}}{\Delta t}$, where $S^{\prime}=\log S$. Hence, we can now write:

$$
k=\frac{1}{\sigma^{2}} \frac{\Delta S^{\prime}}{\Delta t}=\frac{\mu}{\sigma^{2}}+\frac{1}{\sigma} \epsilon \frac{1}{\sqrt{\Delta t}} .
$$

We need to make the remark that $k$ reflects the fact that it is now influenced 
by a random variable, $\epsilon$. We note that $k$ also indicates a dependence on preferences for risk, as expressed by $\mu$. An arbitrage operation is typically carried out on a quite short time scale. Hence, $\frac{1}{\sqrt{\Delta t}}$ will be large, and therefore, the uncertainty multiplied with $\epsilon$ will be large. Therefore, the domain of the amplitude function, $A(k)$ would have a large spread. Using (13) we would need to use a discrete (non-continuous) version of (9), $\frac{1}{\sqrt{2 \pi}} \sum_{j=1}^{\infty} A_{j} \exp \left(i k_{j} x\right)$. A normal pdf is assumed on $\epsilon$. Hence, we can make the argument that a normal pdf is to be assumed on $k$ (as it is now dependent on the random variable $\epsilon$ ). Therefore, we have a normal density function on a set of amplitude functions, $A(k)$. This may allow us to conclude that in that case, the macroscopic uncertainty principle is minimal. It is well known from basic quantum physics that the Fourier transform of a Gaussian is a Gaussian. Since, we assume a normal pdf on $k$ and a density which is normal on $A(k)$, we can assume a normal density on $S^{\prime}$. When a Gaussian is involved it is also widely known from basic quantum physics that the uncertainty relation is minimal.

Note that if the time step is very large, then in (13) one approaches: $\frac{\mu}{\sigma^{2}}$. An argument can be made that over a very large time step, the stock process's derivative towards time is such that it approximates the derivative of the trend. Hence, the return over a very large time step averages out the randomness. We could therefore write that if $\Delta t \rightarrow \infty$, the return should be tending to the risk free rate as randomness is averaged out: $k=\frac{1}{\sigma^{2}} \frac{\Delta S^{\prime}}{\Delta t}=\frac{\mu}{\sigma^{2}}+\frac{1}{\sigma} \epsilon \frac{1}{\sqrt{\Delta t}}=\frac{r_{f}}{\sigma^{2}}$.

\subsection{Arbitrage based risk neutral probabilities derived from $\|\Psi\|^{2}$}

In section 4.1, we mentioned in Claim 12 that the non-arbitrage based risk neutral probabilities could be derived from a zero action functional. However, no such probabilities can be derived from the wave function induced probabilities. As we attempted to explain at the end of section 5.3, this is the case because the zero uncertainty on the momentum, which occurs because of the uniqueness of the risk free rate, induces, via the Fourier formalism, an infinitely widespread density on position. This is unusable as a density.

The pdf induced by the wave function, could potentially be used to describe the risk neutral probabilities, but with arbitrage! Those risk neutral probabilities can then be sourced from a wave function $\Psi$ which belongs to the space $L^{2}(\mathbb{R}, d M)$ of square integrable functions with respect to some measure $M$ on $\mathbb{R}$ such that:

$$
\|\Psi\|^{2}=\int_{\mathbb{R}}|\Psi(S)|^{2} d M(S)<\infty
$$

where $|\Psi(S)|^{2}$ is obtained under the multiplication of $\Psi(S)$ with its conjugate, 
$\Psi(S)^{*}$

Example 22 Let $\Psi(S)=R(S) \exp (i L(S))$, where $R(S)$ and $L(S)$ are some functions of $S$ and $i$ is an imaginary unit. The conjugate of $\Psi(S)$, is: $\Psi(S)^{*}=$ $R(S) \exp (-i L(S))$.

It is important to stress that the wave function in (14) can be interpreted as an 'information wave function'. Although we do not discuss such an interpretation in this paper (see for instance, Khrennikov, 2010; Haven, 2008), it needs to be indicated that if we were to formulate a 'preference for information' function then such device would be intimately related to the notion of probability as it is expressed in (14) above. This is quite different from the interpretation Ma (2010) gives to preferences for information, where such preferences can be unrelated to the probability measure assigned to an event.

\section{6 'Hidden variables' in economics?}

Recall our brief discussion under Definition 13, in section 4.1. of the paper. Using the probability in Definition 13, it is well known (and of course counterintuitive) that the growth rate of a risky asset will be the risk free rate if prob bin $=\frac{\exp \left(r_{f} T\right)-d}{u-d}$ is used. This probability does not refer back to the real event of a stock to go up or down. Haug (2007) calls those 'probabilities of convenience'. It is quite senseful to claim that the expected value of the risky asset when using prob $_{b i n}$ is in effect un-obervable. The expected risky asset price is simply written as $E\left(S_{1}\right)=\operatorname{prob}_{b i n}\left(S_{0} u\right)+\left(1-\operatorname{prob}_{b i n}\right) S_{0} d$, where $S_{0}$ is observable, but $E\left(S_{1}\right)$ is most likely not observable. We wonder whether when following this example, we can not establish a link with the possible existence of hidden variables in a macro-scopic context? Haug (2007) also queries whether the existence of such variables is not natural in an economics setting. Let us explain this point of view a little more.

We can alternatively describe $E\left(S_{1}\right)$ with the real probability (experimentally observable) of the asset to go up in price, $p_{u}$ and the real probability (experimentally observable) of the asset to go down in price, $p_{d}$. However, the value of $E\left(S_{1}\right)$ will most probably be different from $E\left(S_{1}\right)$ when prob $_{b i n}$ is used.

Can we define a relation between $\operatorname{prob}_{b i n}$ and $p_{u}$ and $p_{d}$ ? Let us consider the definition by Jammer (1974) of a hidden variable, as it is used in physics.

Definition 23 Jammer (1974, p. 256-257). In a given theory T about certain physical systems $S$ certain variables $v$ describe the states of $S$; in a theory $T^{\prime}$ about $S$ certain variables $v^{\prime}$... which are not experimentally detectable within the framework of $T$ describe the states of $S$; if the values of $v$...can be obtained 
by some average operation over the values of $v^{\prime}, v^{\prime}$ are called hidden variables (with respect to $T$ ) and $T^{\prime}$ is called a hidden variable theory.

This definition can be actively used in the non-arbitrage theorem (theorem 11) which we covered in section 4 of the paper. Recall the vector of risk-neutral probabilities: $\overrightarrow{\Phi_{\text {prob }}}=\left(\frac{\Phi_{1}}{\Phi_{0}}, \frac{\Phi_{2}}{\Phi_{0}}, \frac{\Phi_{3}}{\Phi_{0}}, \ldots \frac{\Phi_{K}}{\Phi_{0}}\right)$, where each coordinate is a probability and $\Phi_{0}=\exp (-r T)$ is the discount rate (continuously discounted) at the risk-free rate of return, $r$, and $T$ is time. If we are to use $\overrightarrow{\Phi_{\text {prob }}}$ in the calculation of the expected value of an asset then we know those probabilities will most likely not be observed. We can therefore define a probability

vector $\overrightarrow{\Phi_{\text {prob }}^{\prime}}$ which contains the 'real' probabilities to calculate the expected value of the same asset. Clearly, for fixed $\Phi_{0}$, we can establish a pairwise relationship between each coordinate of the vector $\vec{\Phi}=\left(\Phi_{1}, \Phi_{2}, \Phi_{3}, \ldots \Phi_{K}\right)$ and $\overrightarrow{\Phi^{\prime}}=\left(\Phi_{1}^{\prime}, \Phi_{2}^{\prime}, \Phi_{3}^{\prime}, \ldots \Phi_{K}^{\prime}\right)$, in the same way as suggested between $v$ and $v^{\prime}$ in Jammer's definition. The physical system is the payoff of an asset in the future. The states of the payoff are described by $\vec{\Phi}$ and $\overrightarrow{\Phi^{\prime}}$. In that sense, can we say that $\vec{\Phi}$ and $\overrightarrow{\Phi^{\prime}}$ are hidden variables and thereby, $\overrightarrow{\Phi_{\text {prob }}}$ and $\overrightarrow{\Phi_{\text {prob }}^{\prime}}$ are also hidden quantities.

\section{Application 1: The 'likely' arbitrage based price}

Let us consider the argument Ma (2010) makes that the existence of an arbitrage opportunity will lead to the problem that a budget feasible plan will never be most preferred. This very reasonable claim can fit in quite well with the theoretical development we have attempted to sketch in this paper. The development mentioned in section 5.4. of this paper can now be applied. Recall that if there is arbitrage, the uncertainty on the momentum is non-zero and we can use (13): $k=\frac{\mu}{\sigma^{2}}+\frac{1}{\sigma} \epsilon \frac{1}{\sqrt{\Delta t}}$; i.e. to indicate that returns are random. In the case proposed here, the amplitude function $A(k)$ is having as 'envelope' function a normal density (given the random drawings coming from $\epsilon \sim N(0,1))$. Those random returns have underlying random prices which can refer to the existence of 'random' demand functions. Such an argument is not un-reasonable, since in the presence of arbitrage, no definite optimization of utility constrained to a budget is possible (and it is from such optimization that such demand functions are derived).

We can make at least two critical comments on the above development. If such a set of 'random' demand functions can exist why do we have a likely equilibrium price? What is the economics interpretation of the amplitude function, $A(k)$, in that case? The likelihood of an equilibrium price occurring can only be rationalized via the argument that effectively a revealed price can exist when there is arbitrage. An arbitrage based revealed price can exist. Financial 
markets are replete with arbitrage based asset prices. If the returns on an asset are random, as in the case where $k=\frac{\mu}{\sigma^{2}}+\frac{1}{\sigma} \epsilon \frac{1}{\sqrt{\Delta t}}$, then the probability density function over the arbitrage induced equilibriae prices will be a Normal density, since $\epsilon \sim N(0,1)$. If one assumes the returns are random but not with a Normal drawing (which effectively makes $A(k)$ to be not bell shaped in that case), then the functional form of the amplitude function $A(k)$ will need to be rationalized in a slightly different way. The wave functions which are superposed, via the summation operator ${ }^{9}$ (or the integral operator) would indeed echo the existence of arbitrage values (not prices). Those arbitrage values, which are unobservable, would exist on a macroeconomics scale.

We are not alone in the idea of using 'value' as an underlying process to a quoted price of an asset. Already in 2004, Øksendal (2004) did approach this unobserved value/observed actual price formalism within the setting of fractional Brownian motion and the so called 'Wick product' (Duncan et al., 2000).

Figure 2 below shows the situation. The $X$-axis is given by the asset price, $p=S$ and the $Y$-axis by the quantity of the good (indicated by $Q$ ). The $Z$-axis is picturing the time scale, $\Delta t$, upon which arbitrage is exercised. Relatively speaking, the larger is $\Delta t$ the fewer the arbitrage opportunities. One upward supply function is drawn and a variety of downward sloping (random) demand functions are drawn (reflecting thus the non-definite form of those functions given the existence of arbitrage). A probability density function is then drawn over the prices, $p=S$, which, as drawn in the figure, are expressed as part of the coordinates: $\left(S_{1}, Q_{1}\right) ;\left(S_{2}, Q_{2}\right) ;\left(S_{3}, Q_{3}\right)$ and $\left(S_{4}, Q_{4}\right)$. This probability density function is derived from either $\frac{1}{\sqrt{2 \pi}} \sum_{j=1}^{\infty} A_{j} \exp \left(i k_{j} x\right)$ (or the integral form which is given by (9)). Alternatively, the probability density function $P(\{S, r\})=N \exp (-\mathcal{A}(\{S, r\}))$ can be used, when the $\mathcal{A}(\{S, r\}) \neq 0$.

$\overline{9}$ As in the case of $\frac{1}{\sqrt{2 \pi}} \sum_{j=1}^{\infty} A_{j} \exp \left(i k_{j} x\right)$. 


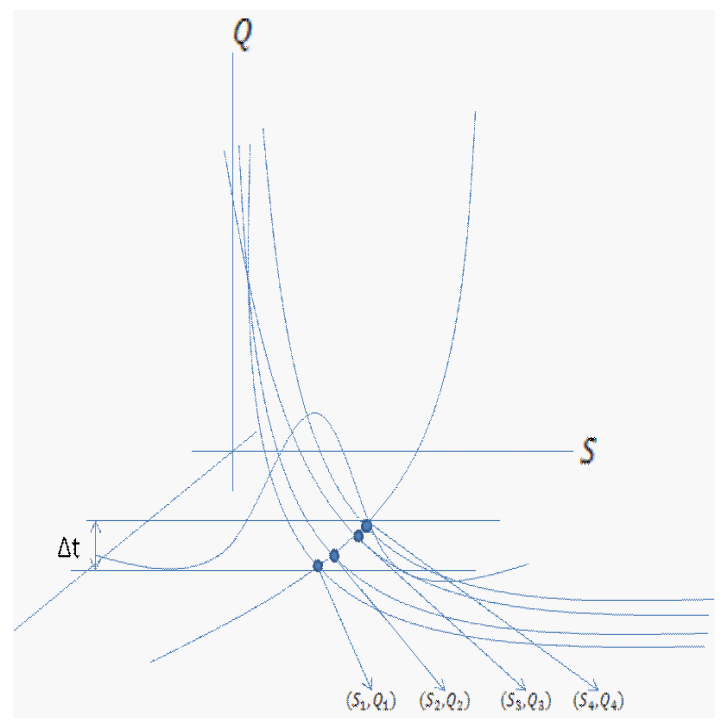

Fig. 2. Arbitrage induced equilibriae

\section{Application 2: Interaction on 'information on returns' versus in- teraction on 'preferences and endowments'}

Föllmer (1974) was one of the first to introduce the idea of random preferences where the randomness depends on the agent's 'environment' (see below). We note that the random economy set up has been followed in other papers.

Föllmer (1974) defines price, $p_{i}$, as an $l$ dimensional positive valued vector, with the restriction that $\sum p_{i}=1$. We use the same notation as in Föllmer (1974).

We can see here that the price can have a probabilistic interpretation. In fact price in this setting can be seen as a state price, as for instance exemplified in theorem 11 above. In Föllmer's random economy model, the state $\omega$ of an agent, $a$, which belongs to a set of agents, $A$, is determined by preferences and the endowment of that agent, $a$. Föllmer (1974) denotes such a state as $\omega(a)$. Given a price $p_{i}$, the excess demand for a good will derive from the usual preference maximization, given the budget constraint which is set by the agent's endowment. The price $p_{i}$, in the words of Föllmer (1974) "induces a well defined individual excess demand..." (p. 55). It should be clear that the price $p_{i}$ is a price which refers to the individual (of agent $a$ 's) excess demand. Hence, the price $p_{i}$ is unobservable. However, in Föllmer (1974) the price $p_{i}$ also induces a well defined "global per capita excess demand" (p.55). Hence, in that case the price $p_{i}$ would be observable. We will come back on this observation below.

In Föllmer (1974) the environment, denoted $\eta$, of agent $a$ is seen as a mapping from the set of all agents (minus the agent $a$ in question) to the set of states, $S$. 
The set of states, $S$, collects all the $\omega($.$) for all agents. We mentioned already$ $\omega($.$) above. A probability value \pi_{a}(s \mid \eta)$ is then defined as the conditional probability an agent $a$ assumes the state $s \in S$ to occur given the environment $\eta$. The conditional probabilities $\pi_{a}(. \mid \eta)=\pi_{a}\left(. \mid \eta^{\prime}\right)$ when both environments $\eta$ an $\mathrm{d} \eta^{\prime}$ coincide on the set of neighbors, $N(a)$, where $a$ is an agent. This set $N(a)$ has a very straightforward definition in Föllmer (1974): $N(a) \equiv$ $\{b \in A:\|b-a\|=1\}$.

The reason why we introduced the notation so far is to consider Föllmer's (1974) idea of a function which would describe the intensity of interaction between agents. For the purposes of this paper, we omit Föllmer's (1974, p.58) discussion of inner-directed states of agents. He defines a function $U(a, b, \omega(a), \omega(b))$, where $a, b \in A$ and $\omega(a), \omega(b) \in S$. It also is indicated that $U\left(a, a^{\prime}, . ..\right)=0$ if $\left\|a-a^{\prime}\right\| \neq 1$ and therefore $U\left(a, a^{\prime}, . ..\right)=0$ if indeed $a^{\prime}$ does not belong to $N(a)$. Föllmer (1974) also indicates that $U\left(a, a^{\prime}, . ..\right)=0$ iff there is no interaction.

For the purposes of this paper we want to consider the interesting definition (albeit slightly altered):

Definition 24 Föllmer (1974, p. 58): $\pi_{a}(s \mid \eta)=$ $\frac{1}{Z(a, \eta)} \exp \left(\sum_{b \in N(a)} U(a, b, \omega(a), \omega(b))\right.$; where $Z(a, \eta)$ is a normalization factor.

The interaction of agents, $a$ and $b$, is measured by the interaction of states (which are given by preferences and endowments), $\omega(a), \omega(b)$. We can assume the set of neighborhoods, $N(a)$ is infinitely large. We can assume, as in Föllmer (1974, p.55) that the set of agents, $|A|=\infty$. If there are infinite amount of agents, $b$, such that $\|b-a\|=1$ then indeed the size of the set $N(a)$ is infinite. We will need the infiniteness of that set, once we want to make the comparison between Definition 24 and our approach.

To come to a comparison with our approach, we need to pose the following question: is an interaction between preferences and endowments comparable to the interaction of information on returns? Or alternatively, how is such interaction between preferences and endowments comparable to the interaction of values of an asset? We will henceforth use 'information on returns' and 'values' interchangeably.

Where is the interaction of 'information' on returns coming from? Consider again, the Fourier integral, $(9): \frac{1}{\sqrt{2 \pi}} \int_{-\infty}^{\infty} A(k) \exp (i k x) d k$. The wave function which pictures 'information' on returns is given by $e^{i k x}$, where $k$ refers to the momentum which echoes return (remark the use of the differential ' $d k$ '). We note that $x$ stands here for the price, $p$. The Fourier integral amalgamates all those information wave functions, $e^{i k x}$. However, the way by which this amal- 
gamation is executed is dependent on the shape of the amplitude function $A(k)$. To express our question slightly differently: does the interaction managed via the amplitude function $A(k)$ of the information wave functions, $\Psi_{1}$; $\Psi_{2} ; \Psi_{3} ; \ldots . \Psi_{\infty}$ (which echo information on returns (i.e. via $k_{j}$ in: $\Psi_{1}=e^{i k_{1} x}$; $\left.\Psi_{2}=e^{i k_{2} x} ; \Psi_{3}=e^{i k_{3} x} \ldots \Psi_{4}=e^{i k_{\infty} x}\right)$ compare to the interaction fuelled by comparing preferences and endowments? If the answer is affirmative, which we believe to be the reasonable approach, then in effect we can compare the probability value derived from Definition 24 with the probability value obtained through the use of the Fourier integral. In other words, we may possibly write:

$$
\begin{aligned}
\pi_{a}(s \mid \eta)= & \frac{1}{Z(a, \eta)} \exp \left(U\left(a, b_{1}\right)+U\left(a, b_{2}\right)+\ldots U\left(a, b_{\infty}\right)\right)= \\
& \int_{v_{2}}^{v_{1}}\left[\frac{1}{\sqrt{2 \pi}} \int_{-\infty}^{\infty} A(k) \exp (i k x) d k\right] \cdot\left[\frac{1}{\sqrt{2 \pi}} \int_{-\infty}^{\infty} A(k) \exp (i k x) d k\right]^{*} d x
\end{aligned}
$$

where the ${ }^{(*)}$ next to the second square brackets indicates the conjugate of the wave function, $\Psi(x)$ is taken. Remark that in the above equation we are effectively claiming that $\pi_{a}(s \mid \eta)$, the conditional probability an agent $a$ assumes the state $s \in S$ to occur given the environment $\eta$, is equal to the probability of finding a certain price $p$. Recall the fact that in a non-arbitrage way, the price $p$ is obtained under preference maximization (given a budget constraint). On prima facie, this would not hold in the above equation since we integrate over a multitude of $k s$; i.e. we use ' $d k$ '. However, we do not necessarily have to constrain ourselves to be in a non-arbitrage price world! We could consider a multitude of subjective prices, and those prices could refer to the value of a good, i.e. each of the $\Psi_{i}$ (echoing information on returns) would also be the value of the good (as opposed to price). It is not so obvious to claim that this multitude of values of a good can occur within the setting of no-arbitrage, since no-arbitrage would imply the existence of no uncertainty on $k$. Remark that in terms of the action functional, the non-zero action functional could then refer to the existence of such multitude of subjective prices for the same good. The zero action functional would then hint to the absence of such multitude of subjective prices.

We could make the argument that the state $s$, which involves preferences and endowments, can translate into a price, but this would be the price the agent ' $a$ ' has in mind. In other words, this price $p$ could possibly be interpreted as a value of an asset. We mentioned in the beginning of this section that the price $p$ could be seen as i) a price inducing excess demand by agent $a$ but it also ii) could induce an excess demand on a per capita basis. In the first case, i), the price is clearly a value, since it is not observed. In the second case, ii), the price is not a value and observable. From a quantum-like perspective, the price, when it appears as value, would be existing in a superposed state (i.e. using the existence of the wave functions $\left.\Psi_{1} ; \Psi_{2} ; \Psi_{3} ; \ldots . \Psi_{\infty}\right)$. Once the wave 
function collapses, we can observe the per capita price. The right hand side of (15) is in fact a superposition of different pieces of information about a return; i.e. a value of an asset. In effect, the right hand side is a superposition of wave functions, by definition.

In summary, does it make sense to compare the left hand side of (15): the probability of the state to occur, with the right hand side of (15): the probability of a value to occur? If the state, which refers to preferences and endowments, does hide value, then this could be reasonable. We note that in Föllmer's (1974) model the price $p_{i}$ is effectively a state price, since $\sum p_{i}=1$. This state price is also intimately connected to the existence of a well defined excess demand. Hence, using the argument by Ma (2010), such price must have a close connection to the existence of non-arbitrage. Surely, state prices are not uniquely tied to the existence of non-arbitrage. Indeed they will also exist when there is arbitrage. We do not even have to be really concerned about the (non) existence of arbitrage, since we may want to think about the availability of a multitude of prices (read: values) for the same good.

In summary, the price, $p_{i}$ may refer to the value of a good. But if the price $p_{i}$ is a state price, then effectively it refers to a state, which in the case of the Föllmer (1974) set up, is the state $s$. We could even claim that since $\sum p_{i}=1$ and $p_{i}>0$, the state price indeed could be a probability. Thus, could $p_{i}=\pi_{a}(s \mid \eta)$ ? That the state price could be seen as the value of an asset, is a quite sensible claim: in effect the state price is non-observed, as would be the 'value' of an asset. Recall the discussion we had in section 5.6 on the nonarbitrage theorem. We mentioned that the states of the payoff are described by $\vec{\Phi}$ and $\overrightarrow{\Phi^{\prime}}$, but those variables are indeed not observed and they clearly are the state prices which can translate into (non-observed) probabilities: $\vec{\Phi}$ and $\overrightarrow{\Phi^{\prime}}$. We can claim that those variables are also hidden variables.

Maybe we can formulate it all as follows. The left hand side of (15) considers how the interaction on preferences and endowments, between agent ' $a$ ' and the rest of the agents (in the set of neighborhoods, $N(a)$ ) leads to a formulaic representation which yields the probability the agent ' $a$ ' assumes the state $s$ to occur. If the state $s$ occurs, then this means there is a certain level of endowment and preference which is revealed. Hence, theoretically, this could thus hide a price which occurs via the procedure of preference maximization given an endowment. This price could be a state price, which coincides with the probability of the state, $s$, occurring. The right hand side of (15) considers how the interaction on the information on returns (read values) between agents leads to a formulaic representation which yields the probability that a good will have a certain value. We assume, via (15), that those two probabilities are the same. The interaction between information on prices (or values), is indeed quite similar to the interaction on preferences and endowments between agents, as the latter hides indeed prices or values. We could opt for 
a two stage sequence where: firstly, i) values exist in a superposed state, as exemplified by (15) and the probability value yielded by $p_{i}=\pi_{a}(s \mid \eta)$ is the individual agent's $a$, price; secondly, ii) the value is revealed as a per capita price. Thus in a first stage, prices are superposed values and the collapsing of the wave function leads to the observed per capita price in stage two.

We make now the following remarks:

- in the no-arbitrage case, $d k=0$ and hence the above comparison, given by (15), would on prima facie be futile (but see below - section 8)

- the right hand side of (15) can be made time dependent. In other words, a dynamic interaction on information functions can be constructed. We note however that the two stage process we mentioned above could also induce some dynamicity (and wave functions could then still remain time independent).

Let us consider some simple examples.

Example 25 Assume that price, $p$, is $x=p$, and let $\Psi(p)=R(p) \exp (i S(p))$; where $R($.$) and S($.$) are two functions. The probability density function found$ by executing: $\left[\frac{1}{\sqrt{2 \pi}} \int_{-\infty}^{\infty} A(k) \exp (i k x) d k\right] \cdot\left[\frac{1}{\sqrt{2 \pi}} \int_{-\infty}^{\infty} A(k) \exp (i k x) d k\right]^{*}$ is then: $|\Psi(p)|^{2}=\exp (2 R(p))$. Let $R(p)=c p$, where $c>0$. Then in this case the probability value: $\int_{v_{1}}^{v_{2}} \exp (2 R(p)) d p=\frac{1}{2 c}\left[\exp \left(2 c v_{2}\right)-\exp \left(2 c v_{1}\right)\right]=\frac{1}{Z} \exp \left(U\left(a, b_{1}\right)+..\right)$, where we have used (14). For simplicity let $Z=2 c=1$, and applying log on both sides of the equation, we get: $2 c v_{2}-2 c v_{1}=U\left(a, b_{1}\right)+U\left(a, b_{2}\right)+\ldots$ or also: $Z \Delta v=U\left(a, b_{1}\right)+U\left(a, b_{2}\right)+\ldots$. Thus, this means that the change in price, $p$, given by $\Delta v$ is given by the interaction of the agent a with agents $b_{i}$. This interaction between those agents is directed from their interaction on endowments and preferences. The change in price given by $\Delta v$ is also the probability of the price to fall between $v_{1}$ and $v_{2}$. This probability is also equal to the probability of the state s to occur, which happens to be equal to a state price.

Example 26 Assume that price, $p$, is $x=p$, and let $\Psi(p)=R(p) \exp (i S(p))$; where $R($.$) and S($.$) are two functions. Let R(p)=\frac{-a}{2} p^{2}, a>0$. The probability of all prices to be between 0 and infinity is: $\int_{0}^{\infty} \exp (2 R(p)) d p=\int_{0}^{\infty} \exp \left(-a p^{2}\right) d p=$ $\frac{1}{2} \sqrt{\frac{\pi}{a}}$, which in an economics setting would require that $a=\frac{\pi}{4}$, as all prices are falling between zero and infinity. We can then write: $\frac{1}{2} \sqrt{\frac{\pi}{a}}=\frac{1}{Z} \exp \left(U\left(a, b_{1}\right)+..\right)=$ 1 . 


\section{A summary of applications 1 and 2}

At the end of application 2, we mentioned that in a first stage, values exist in a superposed state, as exemplified by (15) and the probability value yielded by $p_{i}=\pi_{a}(s \mid \eta)$ is the individual agent's $a$, price. The second stage leads then into a revealed per capita price. So the transition from stage one to stage two means thus, via a quantum mechanical argument, a transition from a superposition of wave functions towards a collapse of a wave function, if there does not exist arbitrage on the revealed price. In other words, if the revealed price refers to an asset which is for instance needed in a arbitrage portfolio, then following the argument by Ma (2010) a budget feasible plan will never be most preferred. We make then the claim, as in section 6 of this paper, that one obtains a set of demand functions (which can be random). In the non-arbitrage case, the 'dual' of this set of demand functions (in the arbitrage case), is just one demand function.

The variation of the parameter $k$ (in the differential operator ' $d k$ ') in this paper, when connected to an economics context, is thus of a double nature: i) in application $1, k$ is varied to denote the momenta of various returns of arbitrage based (unobserved) values (f.i. $k$ is constructed like in (13)) or ii) in application $2, k$ varies to denote the variation of returns of values of a good, across a multiple amount of agents (as to reflect the interaction between agent $a$ and agents $b_{i}$ on the right hand side of (15)). Remark that $k$ stands for the momentum, denoting a return, while the wave function, $e^{i k x}$, echoes the value of the good (either at the macroeconomics level when there is arbitrage or at the microeconomics level where the value is perceived by the individual agent)

Application 2 was concerned in modelling how the superposition of value of a same good (thus for a varying $k$ (returns of values of a good) amongst agents) yields a per capita price for well behaved excess demands. Thus (15), shows this price: $p_{i}=\pi_{a}(s \mid \eta)$. We have assumed that whilst the superposition of values (i.e. non-observed individual prices) is processing, there should be no arbitrage at the value stage. Why? As the value formation must also operate under the idea there is a well behaved excess demand at the individual level. The price $p_{i}$ also induces a well behaved excess demand at the per capita level. Hence, signifying a wave collapse. Application 1 carried this a little a further by assuming that there exist a variety of $p_{i}$ 's because of the possible existence of arbitrage. The variation of $k$ (reflecting variation of return values of a good across many agents (assuming local - or agent based non-arbitrage) is 'metamorphosed' into a variation of $k$ which exemplifies the existence of different returns (on observed prices) appearing because no maximization of utility ever occurs since there is arbitrage. The probability density function which then emerges attempts to pinpoint the most likely price under such a process. 


\section{Conclusion}

Arbitrage can be characterized by a measure of curvature which derives from the theory of fibre bundles. The curvature parameter can be entered into an action functional. The probability value which is a function of this action functional can be connected to risk neutral probabilities as they are used in the non-arbitrage theorem. The Fourier transform can be used to show how a pdf on the amplitude function of wave numbers (they now depend on $\epsilon$ ) yields a pdf from which we can source arbitrage based risk neutral probabilities. We do also show though that the Fourier transform can not be sensibly used in the non-arbitrage case as the pdf is not usable. Via the route of the Fourier transform, we can also introduce some quantum-like ideas in economics. In the applications section of the paper we made the argument that superposed values of a good could be seen to be equal to an unobserved, agent based price. The collapse of a wave function could then yield the per capita based price.

\section{References}

Aerts, D., Broekaert, J., Gabora, L. \& Sozzo, S. (2013). Quantum structure and human thought. Behavioral and Brain Sciences, 36(3), 274-276.

Asano, M., Khrennikov, A., Ohya, M., Tanaka, Y. \& Yamato, I. (2015). Quantum adaptivity in biology: from genetics to cognition. Heidelberg-Berlin-New York: Springer.

Asano, M., Ohya, M., Tanaka, Y., Basieva, I., \& Khrennikov, A. (2011). Quantum-like model of brain's functioning: decision making from decoherence. Journal of Theoretical Biology, 281, 56-64.

Baaquie, B. (1997). A path integral approach to option pricing with stochastic volatility: some exact results. Journal de Physique I, 7 (12), 1733-1753.

Baaquie, B. (2004). Quantum Finance. Cambridge: Cambridge University Press.

Baaquie, B. (2013. Statistical microeconomics. Physica A, 392, 4400-4416.

Baaquie, B. (2014). Path integrals and Hamiltonians: principles and methods. Cambridge: Cambridge University Press.

Basieva, I., Khrennikov, A., Ohya, M., \& Yamato, I. (2010). Quantum-like interference effect in gene expression glucose-lactose destructive interference. Systems and Synthetic Biology 1-10.

Bishop, R.L. \& Crittenden, R.J. (1964). Geometry of Manifolds. Academic 
Press.

Björk, T. \& Hult, H. (2005). A note on Wick products and the fractional Black-Scholes model. Finance and Stochastics, 9(2), 197-209.

Busemeyer, J. R. \& Bruza, P. D. (2012). Quantum models of cognition and decision. Cambridge: Cambridge University Press.

Duncan, T. E., Hu, Y. \& Pasik-Duncan, B. (2000). Stochastic calculus for fractional Brownian motion. SIAM Journal of Control and Optimization, 38, $582-612$.

Dzhafarov, E., Zhang, R. \& Jordan S.M. (2015). Contextuality from quantum physics to psychology. Singapore: World Scientific.

Dzhafarov, E.N. \& Kujala, J.V. (2014). No-Forcing and No-Matching theorems for classical probability applied to quantum mechanics. Foundations of Physics, 44, 248-265.

Dzhafarov, E.N. \& Kujala, J.V. (2012). Selectivity in probabilistic causality: where psychology runs into quantum physics. Journal of Mathematical Psychology, 56, 54-63.

Etheridge, A. (2002). A course in financial analysis. Cambridge: Cambridge University Press.

Föllmer, H. (1974). Random economies with many interacting agents. Journal of Mathematical Economics, 1(1), 51-62.

Frieden, B. \& Hawkins, R. (2010). Asymmetric information and economics. Physica A,389, 287-295.

Harrison, J. M \& Kreps, D. (1979). Martingales and arbitrage in multiperiod securities markets. Journal of Economic Theory, 20, 381-408.

Haug, E. (2007). Derivatives: models on models. New York: J. Wiley.

Haven, E. \& Khrennikov, A. (2013). Quantum social science. Cambridge: Cambridge University Press.

Haven, E., Ma, C. \& Liu, H. \& Shen, L. (2009). Revealing the implied riskneutral MGF with the wavelet method. Journal of Economic Dynamics and Control, 33(3), 692-709

Haven, E. (2008). The variation of financial arbitrage via the use of an information wave function. International Journal of Theoretical Physics, 47, 193-199. 
Haven, E. \& Khrennikov, A. (2016). Statistical and subjective interpretations of probability in quantum-like models of cognition and decision making. Journal of Mathematical Psychology. In Press.

Hawkins, R. \& Frieden, B. (2004). Econophysics. In: science from Fisher information: a unification (Chapter 13). Cambridge: Cambridge University Press.

Hawkins, R. \& Frieden, B. (2012). Asymmetric information and quantization in financial economics. International Journal of Mathematics and Mathematical Sciences. doi:10.1155/2012/470293.

Hawkins, R. \& Aoki, M. \& Frieden, B. (2010). Asymmetric information and macroeconomic dynamics. Physica A, 389, 3565-3571.

Ilinski, K. (2001). Physics of finance. New York: J. Wiley.

Jammer, M. (1974). The philosophy of quantum mechanics. New York: J. Wiley.

Karatzas, I., \& Schreve, S.E. (1998). Methods of mathematical finance. Berlin: Springer

Khrennikov, A. (2010). Ubiquitous quantum structure: from psychology to finance. Berlin: Springer Verlag .

Khrennikov, A. (2015). Quantum version of Aumann's approach to common knowledge: sufficient conditions of impossibility to agree on disagree. Journal of Mathematical Economics, 60, 89-104.

Khrennikov, A. (1999). Classical and quantum mechanics on information spaces with applications to cognitive, psychological, social and anomalous phenomena. Foundations of Physics, 29, 1065-1098.

Khrennikov, A. \& Haven, E. (2016). The Palgrave handbook of quantum models in social science. London: Palgrave MacMillan.

Khrennikov, A. \& Haven, E. (2009). Quantum mechanics and the violations of the sure-thing principle: the use of probability interference and other concepts. Journal of Mathematical Psychology, 53, 378-388.

Kreyszig, E. (1999). Advanced engineering mathematics. New York: J. Wiley. Ma, C. (2010). Advanced asset pricing theory. London: Imperial College Press. Monastyrsky, M. (1993). Topology of gauge fields and condensed matter. Dordrecht: Kluwer Academic Press. 
Neftci, S. (2000). An introduction to the mathematics of financial derivatives. Elsevier.

Øksendal, B. (2004). Fractional Brownian motion in finance. In: Jensen (ed.): Stochastic Economic Dynamics. Cambridge: Cambridge University Press.

Piotrowski, E \& Sładkowski, J. (2003). An invitation to quantum game theory International Journal of Theoretical Physics, 42, 1089-1099.

Piotrowski, E. \& Sładkowski, J. (2004). Quantum game theory in finance. Quantitative Finance, 4, C61-C67.

Plotnitsky, A. (2009). Epistemology and probability: Bohr, Heisenberg, Schrödinger and the nature of quantum-theoretical thinking. Heidelberg-Berlin-New York: Springer.

Plotnitsky, A. (2014). Are quantum-mechanical-like models possible, or necessary, outside quantum physics? Physica Scripta, T163, 014011

Shleifer, A. \& Vishny, R.W. (1997). The limits of arbitrage. The Journal of Finance, 52 (1), 35-55.

Steenrod, N. (1951). The topology of fibre bundles. Princeton: Princeton University Press.

Yukalov, V. \& Sornette, D. (2014). Conditions for quantum interference in cognitive sciences. Topics in Cognitive Sciences, 6, 79-90.

Yukalov, V. \& Sornette, D. (2011). Decision theory with prospect interference and entanglement. Theory and Decision, 70, 283-328 